\title{
Classification neutral to ground (NG) voltage using levenberg-marquardt neural network (LMNN)
}

\author{
Suzaryfazli Kamaruddin, Ahmad Farid Abidin, Mohd Abdul Talib Mat Yusoh \\ Faculti of Electrical Engineering, Universiti Teknologi MARA (UiTM), Malaysia
}

\begin{tabular}{l}
\hline \hline Article Info \\
\hline Article history: \\
Received Mar 30, 2019 \\
Revised Jun 11, 2019 \\
Accepted Ju1 28, 2019 \\
\hline
\end{tabular}

\section{Keywords:}

Neural network (NN)

Neutral to ground (NG) voltage

Power quality (PQ)

Wavelet transform (DWT)

\begin{abstract}
In electrical systems nowadays, power quality issues have become a major concern for customers and electrical utilities. The high Neutral to ground (NG) voltage are one of the power quality issues which could cause adverse effect such as malfunction the devices, neutral overheating and electricity shock. Thus, the high NG voltage should be classified in order to perform the mitigation work accurately. This paper presents the classification of neutral to ground voltage using Levenberg-Marquardt Neural Network (LMNN) technique. The Discrete Wavelet Transform (DWT) is applied in this method to extract the features of NG voltage which needed in classification process. The result shows the LMNN perform accurately in classify the types of NG voltage, where its accuracy result is reach more than $90 \%$ accuracy.
\end{abstract}

Copyright $\odot 2019$ Institute of Advanced Engineering and Science. All rights reserved.

\section{Corresponding Author:}

Ahmad Farid Abidin,

Faculti of Electrical Engineering, 40450 Shah Alam, Selangor, Malaysia.

Email: ahmad924@salam.uitm.edu.my

\section{INTRODUCTION}

Power industry nowadays is concern about power quality issues because of the customers demand. The typical PQ disturbances are voltage sags, transient, harmonics, and voltage swells. This disturbance can affect to the economical losses for industry such as the quality of the industry products [1]. Many types of equipment nowadays are using power electronics and more sensitive to this kind of disturbances where the damage gives high impact in terms of cost [2]. Power quality is referred how near the voltage waveform being sinusoidal at rated magnitude and frequency [3]. Voltage sags and swells are the most common types of power quality disturbances that familiar has been discussed [4]. Most of the discussed disturbance are occur at phase connection. However, some study shows that such disturbance also could occur at neutral to ground connection [5-8]. There are some factors that cause high NG voltage. One of the factors is the phase current imbalance that caused by a large number of single phase loads applied to one phase elsewhere [9]. The ground connection plays a big part to this event, where poor earth/ground connection at local ground point or at the substation can affect the NG voltage.

Based on the corresponding problems, the types of high NG voltage should be classified where the corrective work can perform on right place. In this case, the data preprocessing is utilized in order to get a better model for classification of the NG voltage. There are a number of methods which being used for data preprocessing of voltages signals. Visual inspection method is used in the past and it is seemed not practical nowadays where the voltage signals involve a large number of data [10]. This method required someone who has a good knowledge on how to gain desired information from the voltage signals. In the past studies, there are modern methods such as fractal based method, S-transform (ST) method, Fast Fourier Transform (FFT) method, Short Time Fourier Transform (STFT) method and Discrete Wavelet Transform (DWT) [11-14].

In this paper, wavelet transforms (WT) method is proposed for data preprocessing methods and artificial neural network is proposed for classification of the NG voltage. This method can apply various 
lengths of windows according to the amount of signal frequencies [15]. WT can analyze PQ disturbance simultaneously in both time and frequency domains [16-18]. For classification of the power quality disturbances, Artificial Neural Network (ANN) method is widely use in the past studies. ANN has the ability to classify noise data in the large number of data [16]. ANN has the ability to implicitly detect complex nonlinear relationships between dependent and independent variables. It is also having the ability to detect all possible interactions between predictor variables and has the availability of multiple training algorithms. The ANN model developed based on Levenberg Marquadt as proposed by [19] was used in order to classify the high NG voltage component. The Levenberg Marquardt algorithm which known as LMNN has chosen due to the shown better performance in terms of accuracy as mentioned by [20].

\section{RESEARCH METHOD}

\subsection{Data Preparation}

There are two types of NG voltage component that have been analyzed and need to be classified in this study. One of these high NG voltage components is due to harmonics where this component is originated from nonlinear load. Another high NV is known as transient which due to the starting load or fault. In this paper, 9 event of high NG voltage are recorded using Fluke Analyzer in 9 different places. Figure 1 shows all the 9 NG voltage signal.
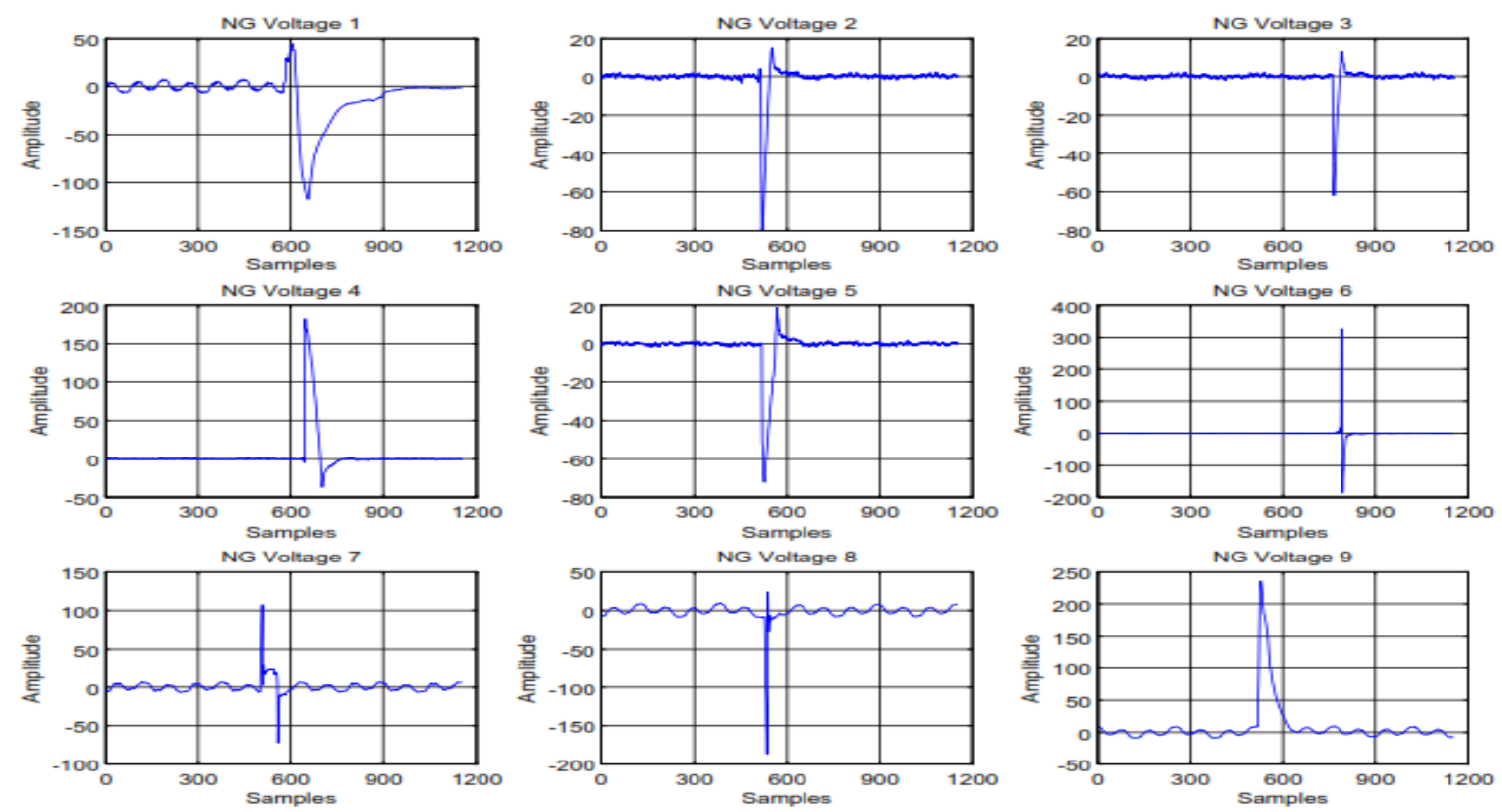

Figure 1. All 9 NG voltage signals

In order to get the best classification result, feature extraction need to be chosen correctly. In this paper, the WT based on Discrete Wavelet Transforms (DWT) is used in order to extract the information. DWT provides sufficient information with less computation time sufficiently. DWT can be expressed as;

$D W T \psi x(j, k)=\int_{-\infty}^{\infty} x(t) \psi_{j, k} d t$

where, $\psi_{j, k}(t)=\frac{1}{\sqrt{2^{j}}} \psi\left(\frac{t-k 2^{j}}{2^{j}}\right)$

Where, $\mathrm{j}$ and $\mathrm{k}$ are the integers of scale parameter and shift parameter respectively. ${ }_{j, k}(t)$ is the mother wavelet. 
The choices of mother wavelet plays significant role where higher scale signal decomposition is needed [3]. In order to decompose the NG signal, the Daubechies (db) wavelet technique is implemented. From the db wavelet family, forth order of Daubechies (Daub4) is chosen in this paper as it is the simplest one where Daub4 is similar as the Haar transform. Daub4 is the most localized, i.e. compactly supported, in time [21]. From Daub4 wavelet transform, the mean absolute deviation (MAD) and standard deviation (SD) at level 4 are chosen as the input for two types of high NG voltage component. MAD is the average absolute deviation of a data set from a central point and SD is a measure of how spread out a distribution. In other words, they are measures of variability.

\subsection{Classification Stages}

The LMNN is a very simple technique but enable to gives the performance of result with high accuracy. The LMNN is a powerful tool that frequently to be used in many applications in term of solving generic curve-fitting problems. Thus, in this case, the LMNN is utilized to classify the types of NTEV rise in electrical system.

In order to obtain best performance of classifier, the required training parameters of this LMNN such as learning rate, momentum rate and hidden layer must be optimized first. Figure 2 show the inputs and outputs for classification of high NG voltage.

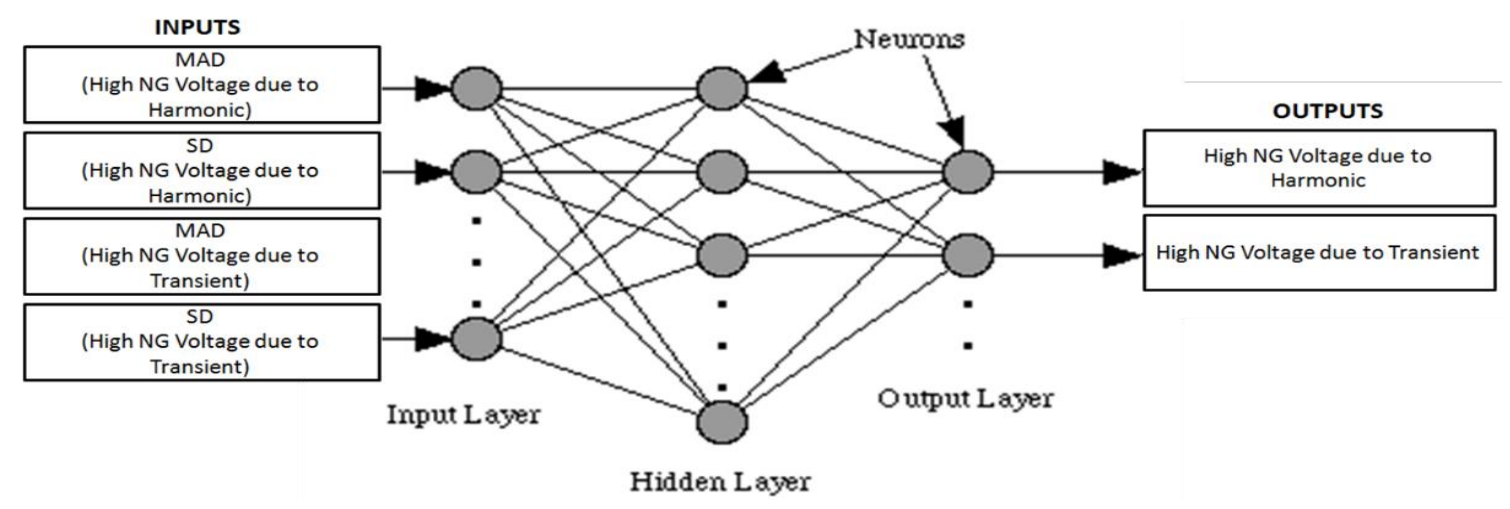

Figure 2. The inputs and outputs for classification of high NG voltage

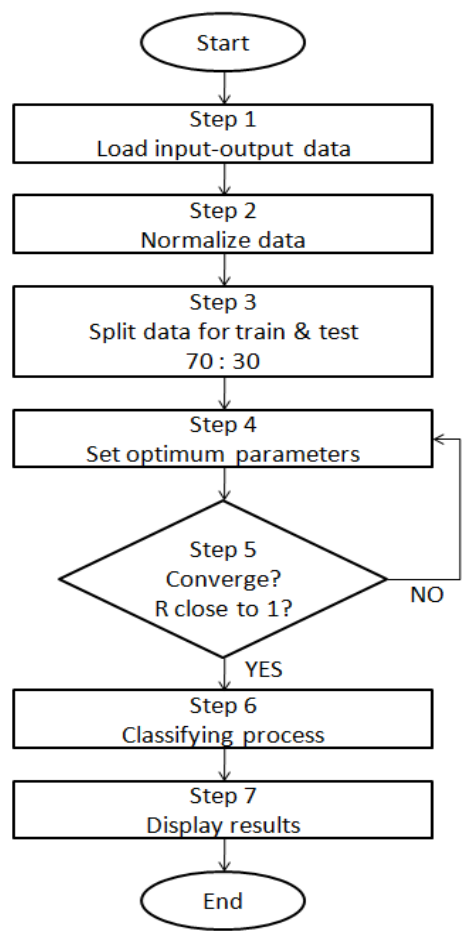

Figure 3. The flow chart for classification of high NG voltage 
Figure 3 shows seven steps need to be implemented for classification of high NG voltage. First step involves the conversion of data from the wavelet transform into MATLAB file. For the next process, the data were imported into the MATLAB. The values of data must be in a small number or in the specified range. This lead to the second steps, where data normalization must be done first so that the values of the data are more appropriate to be an input for the ANN. Training and testing process are the most important for ANN. For the third step, dividing the data into two group by $70 \%$ of the data are used in training process and the other $30 \%$ are used in testing process. For better classification results, the training data is not used for the testing process. This data is clustered into train input, train target, test input and test target using a MATLAB script.

In this paper, the data is modeled by 54 numbers of data which is consist 45 numbers of data from high NG voltage due to harmonic and 9 numbers of data from high NG voltage due to transient. By using $70: 30$ ratio, the numbers of data that used in training process is 38 and 16 numbers of data is use for testing purposes. Optimization process of the required training parameters are in the step number 4. Learning rate and momentum rate are in the range of 0 to 1 and it is varies from 0.1 to 1 with increment of 0.1 . For learning rate optimization, momentum rate is set to its maximum value which is 1 and the hidden layer is set to 10 . By comparing the mean square error (mse) from 0.1 to 1 , the lowest mean square error from the increment is chose as the optimal values for learning rate. The optimal values for learning rate is then used to determine the momentum rate. Same step is applied to get the optimal momentum rate. The optimal values of the hidden layer then are determined using both optimal values of the learning rate and momentum rate. The error goal is set to 0.01 and epochs are set to 100. All of these parameters are used for training and testing process. The optimize parameters of this paper is shown in Table 1.

Table 1. The Optimize LMNN Parameters

\begin{tabular}{cc}
\hline Parameters & Optimal Value \\
\hline Nodes in input layer & 4 \\
Noutput layer size & 3 \\
Number of nodes in hidden layer $(\mathrm{hl})$ & 0.1 \\
Learning rate (lr) & 0.2 \\
Momentum rate $(\mathrm{mr})$ & 9 \\
Epochs & 100 \\
Error goal & 0.01 \\
\hline
\end{tabular}

Step 5 involves the decision process of the LMNN. To proceed to the next step, the system need to achieve the converge state during the training stage. The system will return to the previous step if the converge state and regression value do not met the given criteria. Step 6 is the classifying process stage for training and testing. All the high NG voltage for both training and testing will be separated into their own group either high NG voltage due to harmonic or to high NG voltage due to transient. In order to separate these two types of outputs, a threshold is set in range of value 1 to 2.1 .5 is the threshold value that is set in this LMNN model. This threshold will classify what group is the high NG voltage belongs to. As for the final step, the prediction and classification results are display in graph.

\section{RESULTS AND ANALYSIS}

Using MATLAB R2011a version, the high NG voltage signal were analysed using wavelet toolbox. The signal that represents the data is then sampled into certain period of time before it was analysed using One-Dimensional Discrete Wavelet Analysis Tool. By using the toolbox, the forth order Daubechies (Daub4) was chose and the data were analysed by using decomposition from level 1 to level 5 . This analysed process is done repeatedly and properly in order to get the best feature extraction from the signal where it is used as the inputs for the ANN. From the analysis process, it is shown the Daub4 decomposition at level 4 has significant value for all data. The details decomposition at level 4 is chose for statistical analysis and the Median Absolute Deviation (MAD) and Standard Deviation (SD) values are used as the inputs for the ANN model.

Figure 4 (a) and (b) shows the decomposition from level 1 to 5 for high NG voltage due to harmonic and transient respectively.

There are total 2 inputs and 2 outputs for this LMNN. There are 38 numbers of data is used for the training process and 16 numbers of data is used for the testing process. From the simulation of the ANN, the best of training performance was record at epochs 1 with the value of 0.0093067 . The value of regression (R) is 0.97469 (almost 1) which indicate that the LMNN is in a good performance. 
Figure 5 (a) and (b) shows the simulation result for best training performance of the LMNN and the regression of the ANN respectively.

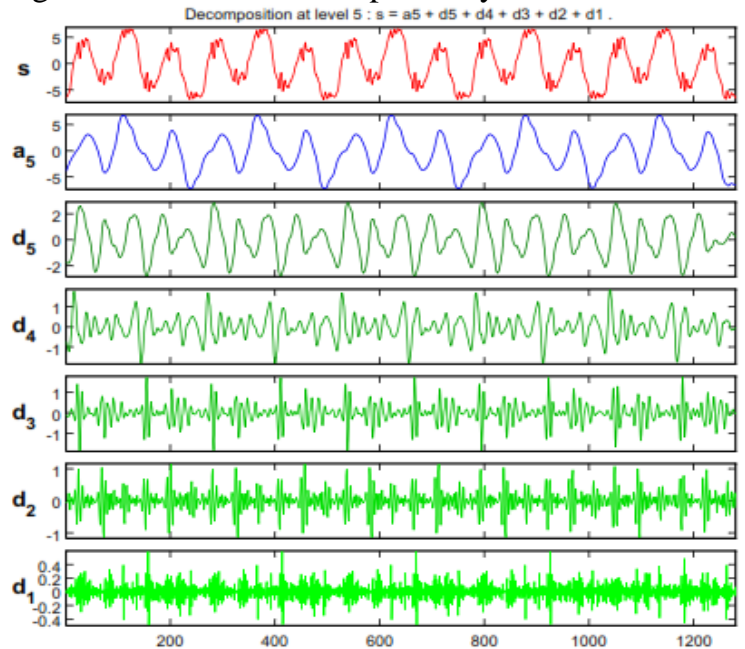

(a)
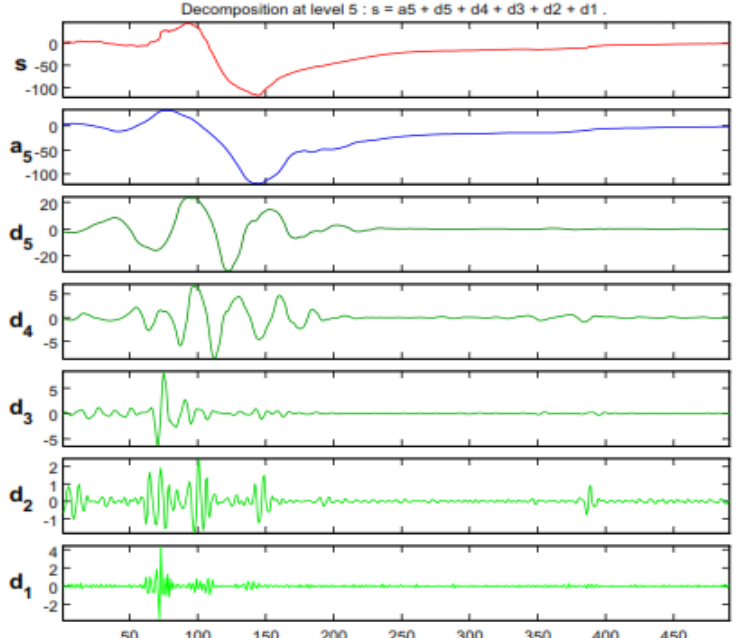

(b)

Figure 4. The decomposition from level 1 to 5 for high NG voltage due to (a) harmonic (b) transient

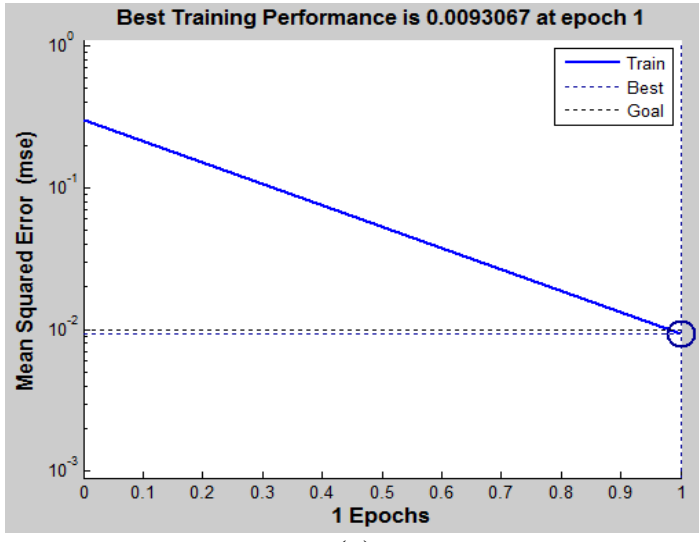

(a)

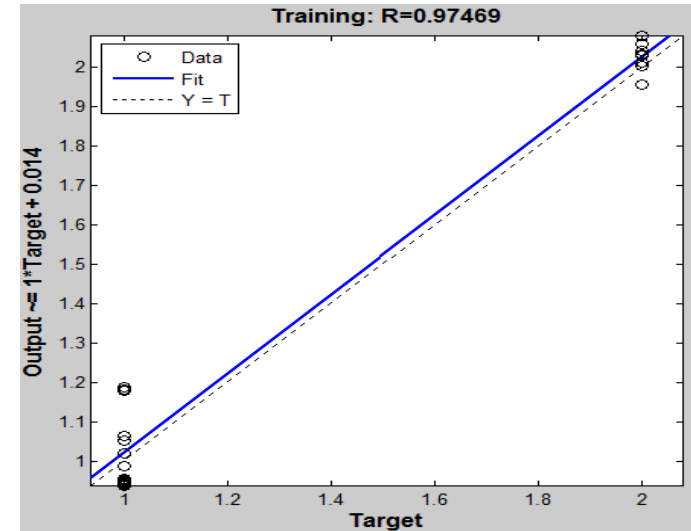

(b)

Figure 5. Training result (a) the best training performance (b) regression

The result of the prediction and the classification of training and testing data are shown in Figure 6 (a) and (b).
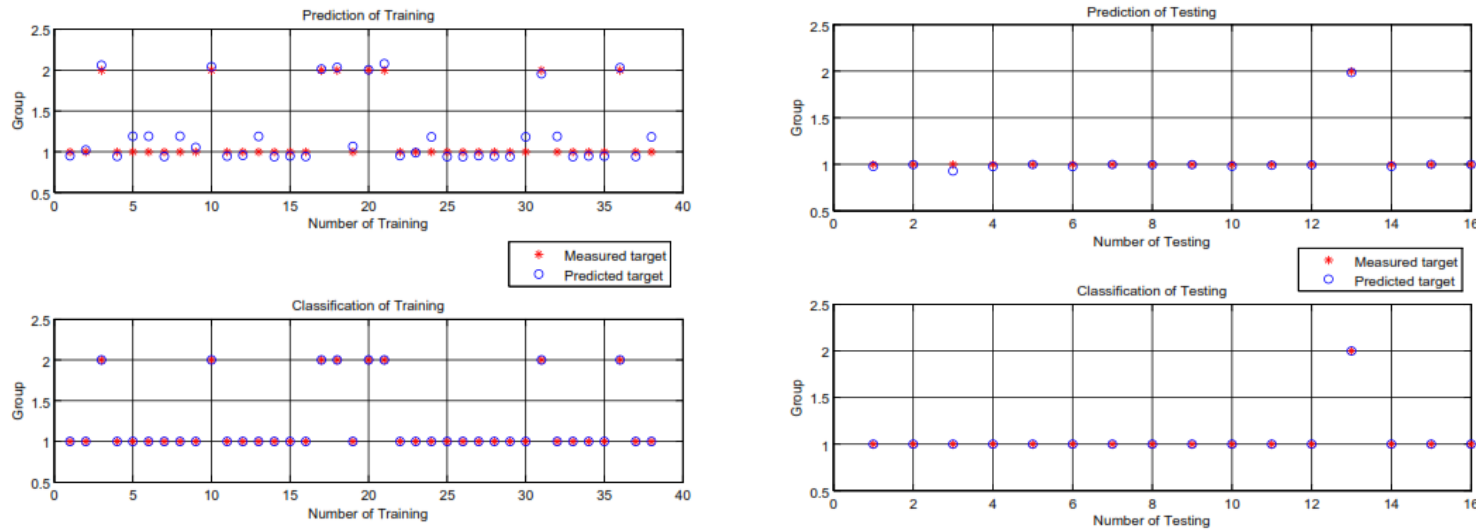

Classification neutral to ground $(N G)$ voltage using levenberg-marquardt... (Suzaryfazli Kamaruddin) 
(a)

(b)

Figure 6. The prediction and classification of (a) training (b) testing

From the prediction of testing graph, it is shown that all the values of data are nearly fall to its respective group. The accuracy of the prediction for training and testing is then calculated. Table 2 show the value of error and the accuracy for both training and testing.

Table 2. Testing Prediction Error

\begin{tabular}{cccc}
\hline Test Predict & Test Output & Error & Accuracy $(\%)$ \\
\hline 0.97644 & 1 & -0.02356 & 97.64 \\
0.99644 & 1 & -0.00356 & 99.64 \\
0.92663 & 1 & -0.07337 & 92.64 \\
0.97479 & 1 & -0.02521 & 97.48 \\
0.99073 & 1 & -0.00297 & 99.70 \\
0.97550 & 1 & -0.02450 & 97.55 \\
0.99831 & 1 & -0.00169 & 99.83 \\
0.99500 & 1 & -0.00500 & 99.50 \\
0.99560 & 1 & -0.0040 & 99.56 \\
0.97859 & 1 & -0.02141 & 97.86 \\
0.98839 & 1 & -0.01161 & 98.84 \\
0.99253 & 1 & -0.00747 & 99.25 \\
1.98476 & 2 & -0.01524 & 99.24 \\
0.97584 & 1 & -0.02416 & 97.58 \\
0.9969 & 1 & -0.00031 & 99.97 \\
0.99830 & 1 & -0.00170 & 99.83 \\
\hline
\end{tabular}

From Table 2, there are 15 numbers of data that are classified into group 1 and only 1 number of data classified into group 2. The table shows the accuracy of the prediction for testing of the LMNN are all $90 \%$ and above for all data. The mean square error that obtains from testing process 0.000544 . This is indicated the good performance of the LMNN model and it make perfect classification for the two desired outputs.

\section{CONCLUSION}

This paper has presented the classification of high NG voltage due to the harmonic and transient using ANN. In this paper, the LMNN classifier has successful developed to perform the classification of NG voltage accurately. The result shows that the accuracy of testing prediction error is $90 \%$ and above. Further, the mean square error that obtains from the testing process is small, where it value is 0.000544 . The value of the accuracy and the mean square error are acceptable for the classification of the neutral to ground voltage components. Thus, it concluded that the LMNN classifier able to perform a high accuracy in classifying the types of NG voltage.

\section{ACKNOWLEDGEMENTS}

The author acknowledges the financial support given by Ministry of Higher Education (MOHE) Malaysia for sponsoring this research in the form of grant-in-aid 600-RMI/FRGS 5/3 (0103/2016).

\section{REFERENCES}

[1] V. Ignatova, P. Granjon, and S. Bacha, "Space vector method for voltage dips and swells analysis," IEEE Trans. Power Deliv., vol. 24, no. 4, pp. 2054-2061, 2009.

[2] J. Douglas, "Power quality solutions," IEEE Power Eng. Rev., vol. 14, no. 3, pp. 3-7, 1994.

[3] M. Tuljapurkar and A. A. Dharme, "Wavelet Based Signal Processing Technique for Classification of Power Quality Disturbances,” 2014 Fifth Int. Conf. Signal Image Process., pp. 337-342, 2014.

[4] K. Daud, A. F. Abidin, A. P. Ismail, M. D. A. Hasan, M. A. Shafie, and A. Ismail, "Evaluating windowing-based continuous S-transform with neural network classifier for detecting and classifying power quality disturbances," vol. 13, no. 3, pp. 1136-1142, 2019.

[5] W. Xu, J. R. Acharya, R. Torquato, and J. Yong, "A Method to Determine Stray Voltage Sources-Part I: Concept and Theory," IEEE Trans. Power Deliv., vol. 30, no. 2, pp. 713-719, Apr. 2015.

[6] M. J. E. Alam, K. M. Muttaqi, and D. Sutanto, "Alleviation of Neutral-to-Ground Potential Rise Under Unbalanced Allocation of Rooftop PV Using Distributed Energy Storage," IEEE Trans. Sustain. Energy, vol. 6, no. 3, pp. 889- 
898, Jul. 2015.

[7] H. Liu and Y. Tan, "Brief Analysis on Reasons and Solutions of Excessive Neutral-Earth Voltage in Machine Room," in Proceedings of the 2013 International Conference on Advanced Information Engineering and Education Science (ICAIEES 2013), 2013.

[8] M. A. T. M. Yusoh, S. H. Asman, Z. M. Yasin, and A. F. Abidin, "Classification of the NTEV problems on the commercial building," Indones. J. Electr. Eng. Comput. Sci., vol. 9, no. 2, 2018.

[9] H. W. Davis, "Distribution Systems," pp. 73-86, 1950.

[10] M. M. S. Lira, R. R. B. De Aquino, A. A. Ferreira, M. A. C. Jr, A. B. Carlos, and O. Lira, "Improving Disturbance Classification by Combining Multiple Artificial Neural Networks," pp. 3436-3442, 2006.

[11] S. J. Huang and J. M. Lin, "Application of box counting method-based fractal geometry technique for disturbance detection in power systems," Power Eng. Soc. Gen. Meet. 2003, IEEE, vol. 3, no. 2, pp. 1604-1608, 2003.

[12] Z. Liu, Y. Cui, and W. Li, "Combined power quality disturbances recognition using wavelet packet entropies and S-transform,” Entropy, vol. 17, no. 8, pp. 5811-5828, 2015.

[13] J. Barros and R. I. Diego, "A review of measurement and analysis of electric power quality on shipboard power system networks," Renew. Sustain. Energy Rev., vol. 62, pp. 665-672, 2016.

[14] O. P. Mahela, A. G. Shaik, and N. Gupta, "A critical review of detection and classification of power quality events," Renew. Sustain. Energy Rev., vol. 41, pp. 495-505, 2015.

[15] S. Santoso, E. J. Powers, W. M. Grady, and A. C. Parsons, "Power quality disturbance waveform recognition using wavelet-based neural classifier-part 1: Theoretical foundation," IEEE Trans. Power Deliv., vol. 15, no. 1, pp. 222$228,2000$.

[16] P. Kanirajan and V. Suresh Kumar, "Power quality disturbance detection and classification using wavelet and RBFNN," Appl. Soft Comput., vol. 35, pp. 470-481, 2015.

[17] K. Thangaraj, N. P. Subramaniam, R. Narmada, and O. M. M, "Analysis of harmonics using wavelet technique," vol. 9, no. 3, pp. 1669-1675, 2019.

[18] S. C. Shekar, G. R. Kumar, E. Engineering, E. Engineering, and A. Info, "A transient current based micro-grid connected power system protection scheme using wavelet approach," vol. 9, no. 1, pp. 14-22, 2019.

[19] Z. Ming, W. Jian-Ru, W. Zhi-Qiang, and C. Jian, "Control Method for Power Quality Compensation Based on Levenberg-Marquardt Optimized BP Neural Networks," 2006 CES/IEEE 5th Int. Power Electron. Motion Control Conf., pp. 1-4, 2006.

[20] D. Batra, "Comparison Between Levenberg-Marquardt And Scaled Conjugate Gradient Training Algorithms For Image Compression Using MLP,” no. 8, pp. 412-422, 2014.

[21] S. Santoso, E. J. Powers, W. M. Grady, and P. Hofmann, "Power quality assessment via wavelet transform analysis," IEEE Trans. Power Deliv., vol. 11, no. 2, pp. 924-930, 1996.

\section{BIOGRAPHIES OF AUTHORS}

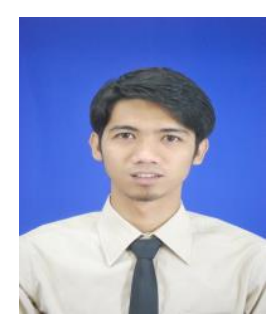

Suzaryfazli Kamaruddin was born in Malaysia on Octobor 26, 1986. He received his Bachelor of Electrical Engineering (Hons.) from Universiti Teknologi MARA (UiTM) in 2016. He is currently enrolled as an Engineer in Empire MABS Resources.

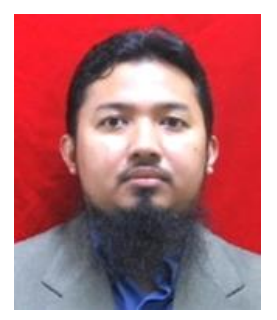

Ahmad Farid Abidin was born in Malaysia, on Dec 25, 1978. He received his Bachelor of Engineering in Electrical, Electronic, and System Engineering from Universiti Kebangsaan Malaysia (UKM), M. Sc in Electrical Engineering from Universiti Teknologi MARA (UiTM), and Ph.D degree from Universiti Kebangsaan Malaysia (UKM), in 2000, 2005, and 2011, respectively. He is currently a lecturer and Head of Centre for Electrical Power Engineering Studies (CEPES) in Universiti Teknologi MARA (UiTM) Shah Alam. His main research interests are in power quality and power system protection.

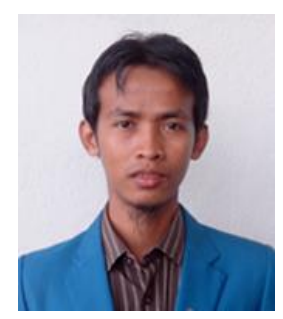

Mohd Abdul Talib Mat Yusoh was born in Malaysia, on August 24, 1988. He received Bachelor of Electrical Engineering (Hons.) and Master of Electrical Engineering from Universiti Tun Hussein Onn Malaysia, (UTHM) in 2009 and 2012 respectively. He is currently enrolled as a $\mathrm{PhD}$ student at Universiti Teknologi MARA, (UiTM). 